\title{
There is no such thing as a free lunch
}

Von R. Iten, R. Maggi, H. Schelbert-Syfrig und A.J. Zimmermann, Universität Zürich

Eine Replik auf die Anmerkung von St. Felder in der

Schweizerischen Zeitschrift für Volkswirtschaft und Statistik, Heft 2/1989

St. Felder kritisiert in dieser Anmerkung zwei Beiträge von $H$. SchelbertSyfrig/A. Zimmermann und von H. Schelbert-Syfrig/Th. Lang/I. Buse/J. Henzmann/R. Maggi/R. Iten/C. Nielsen, die sich mit der Waldwirtschaft und deren positiven Externalitäten (insbesondere Erholungsfunktion und Schutzfunktion) beschäftigen. Seine Kritik bezieht sich auf die in den beiden Beiträgen erhobene Forderung, wonach die positiven Externalitäten der Waldwirtschaft zu internalisieren seien.

Gemäss seinem Argument ist eine Internalisierung nicht erforderlich, wenn die betreffende Externalität nicht knapp ist. Da im Falle der Erholungsfunktion des Waldes die Knappheit empirisch nicht gesichert sei, beurteilt er die Forderung nach Abgeltung der Erholungsfunktion als problematisch. Felder stützt seine Argumentation durch ein formales Modell der waldwirtschaftlichen Produktion mit der Waldfläche als Aktionsparameter, wobei die Dienstleistungsfunktionen als «Gratis»-Kuppelprodukte anfallen. Es stellt sich hier die grundsätzliche Frage, ob es tatsächlich Güter gibt, die gratis anfallen.

Das Modell muss als äusserst schlecht gewählt bezeichnet werden, wenn die von den kritisierten Autorinnen und Autoren untersuchte Fragestellung im Auge behalten werden soll. In den beiden Studien wird der Frage nachgegangen, ob im Bereich der Waldwirtschaft aufgrund externer Effekte mit Fehlallokationen zu rechnen sei. Dabei wurde nicht nach Fehlallokationen bezüglich der Waldfläche gesucht. Seit über 100 Jahren versucht der Staat mit den im Forstgesetz verankerten Rodungsverbot und Prinzip der Nachhaltigkeit, die Waldfläche zu erhalten. Bezeichnenderweise waren externe Effekte (Naturkatastrophen, Lawinen und Überschwemmungen aufgrund der Übernutzung der Wälder) Grund für die Einführung. Eine Berücksichtigung der Waldfläche als exogene Grösse ist deshalb bei Untersuchungen zur Waldwirtschaft naheliegend.

Untersucht wurde, ob mit Fehlallokationen im Bereich der Waldnutzung und damit -pflege des heutigen (flächenmässigen) Waldbestandes zu rechnen sei. Dies geschah vor dem Hintergrund der im Beitrag von Schelbert/Zimmermann geschilderten Probleme der Wald- und Holzwirtschaft. Dort wird gezeigt, dass die schweizerischen Wälder unternutzt werden, weil die durch die internationale Konkurrenz gegebenen Holzpreise die Produktionskosten an vielen Standorten nicht mehr decken. Bleibt diese Situation bestehen, wird sich die Qualität 
der Schweizer Wälder infolge der ausbleibenden Nutzung stark verschlechtern (Überalterung und als Folge grossflächige Zusammenbrüche), und die Wälder können bald einmal die erwähnten Dienstleistungsfunktionen nicht mehr wahrnehmen.

Dieses Szenario der Wälder mag illustrieren, dass sich die heute relevante waldwirtschaftliche Allokationsfrage nicht auf die eingesetzte Waldfläche, sondern auf die optimale Nutzung und damit Pflege des Waldes bezieht. Die Frage nach der optimalen Nutzungsintensität steht im Zentrum des öffentlichen Interesses. Trotzdem wird sie im Modell von Felder zum vornherein eliminiert, da es von einer konstanten Holzproduktion pro Flächeneinheit ausgeht. Das Argument der «Nichtknappheit» der Dienstleistungsfunktionen in der von Felder vorgebrachten Form ist deshalb nicht von Belang.

Es ist in diesem Zusammenhang zu beachten, dass bei der waldwirtschaftlichen Produktion Holz und Dienstleistungen keine vollständigen Kuppelprodukte darstellen. Ein bezüglich der Dienstleistungsfunktionen optimaler Wald weist eine Nutzungsintensität auf, die zwischen den zwei Extremen «Urwald» und «Holzplantage» liegt. Bei der Bewirtschaftung der Wälder besteht entsprechend ein Trade Off zwischen Holzproduktion und Produktion von Dienstleistungen. Diese in der Realität bestehende Nutzungskonkurrenz steht im Widerspruch zu den Annahmen des Modells von Felder.

Sie hat aber Konsequenzen für die Analyse der Produktionsbedingungen der Waldwirtschaft. Es zeigt sich, dass der Waldwirtschaft wegen den Auflagen im Forstgesetz zusätzliche Produktionskosten anfallen, die abgegolten werden müssen.

Der Umstand, ob auf der Nachfragerseite Knappheit vorliegt, hat einen Einfluss auf die Art und Weise einer allfälligen Internalisierung. Falls im Bereich der Walderholung Knappheiten in Form von "Verstopfungen» von sich gegenseitig behindernden Waldgängern auftreten, wäre das ein Hinweis auf Externalitäten auf der Nachfragerseite, die zu internalisieren wären. Sind auf der Nachfragerseite keine solchen Phänomene zu beobachten, so ist diesbezüglich auch keine Internalisierung notwendig. Es handelt sich in diesem Fall um eine positive Externalität mit Kollektivgutcharakter. Der optimale Preis für den Nachfrager ist in diesem Fall null, weil ein Ansteigen der Zahl der Nachfrager weder Kosten noch Nutzen verursacht. Die Nutzung des Waldes als Erholungsraum erhöht jedoch die Kosten der Holzproduktion bei der Holzernte und Verjüngung. Diese externen Kosten der Walderholung werden bis heute nur teilweise abgegolten.

Dies bedeutet, dass eine Internalisierung bzw. Abgeltung der gegenüber Dritten erbrachten Dienstleistungen auf der Produzentenseite notwendig ist. Bedingung für eine gesamtwirtschaftlich optimale Nutzung und Pflege des Waldbestandes und damit optimale Waldqualität ist in diesem Fall eine Pigousche Abgeltung für 
die Waldwirtschaft als Erzeugerin der positiven Externalität. Theoretisch sollte sich dabei die Abgeltung auf den sozialen Grenznutzen, den die Outputeinheiten der Externalität stiften, beziehen. Im Falle der Erholungsfunktion ist eine direkte Messung des physischen Outputs nicht möglich. Als sinnvolle Näherungsgrösse können die Anzahl Besuche in einem Wald verwendet werden. Als weitere Grundlage für die Abschätzung der optimalen Subventionshöhe muss der Grenznutzen der Waldbesuche bekannt sein. Dieser kann z. B. durch Befragung der Waldbesucher erfasst werden.

Die von Felder kritisierten Arbeiten entsprechen dieser Logik. Während in der ersten Arbeit vor allem auf die Existenz von positiven Externalitäten und deren Auswirkungen auf die Waldwirtschaft aufmerksam gemacht wird, befasst sich die zweite Studie mit der empirischen Erfassung dieser Externalitäten. Dies gelang auf der Basis unterschiedlicher Methoden.

Die Kritik von Felder basiert auf einem Modell, das im Zusammenhang mit der von den Autorinnen untersuchten Fragestellung nicht anwendbar ist. Sie kann bestenfalls als Hinweis darauf aufgefasst werden, bei einer zukünftigen Studie der Einordnung der gewählten Fragestellung noch mehr Beachtung zu schenken.

\section{Literatur}

Baumol, W.J./Oates, W.E. (1975): The Theory of Environmental Policy, Externalities, Public Outlays, and the Quality of Life, Kap. 3 und 4. Englewood Cliffs, New Jersey.

Buchanan, J.M./Stubblebine, C. (1962): Externality. Economica, 371-384.

Felder, St. (1989): Sind Externalitäten in jedem Fall zu internalisieren? Schweizerische Zeitschrift für Volkswirtschaft und Statistik 125, 189-193.

Schelbert-Syfrig, H./Lang, Th./Buse, I./Henzman, J./Maggi, R./Iten, R./Nielsen, C. (1988): Wertvolle Umwelt. Schriftenreihe Wirtschaft und Gesellschaft der Zürcher Kantonalbank, September 1988.

Schelbert-Syfrig, H./Zimmermann, A.J. (1988): Konkurrenz und Umweltschutz. Wald- und Holzwirtschaft zwischen Ökonomie und Ökologie. Schweizerische Zeitschrift für Volkswirtschaft und Statistik 124, 289-302. 